\title{
Knowledge, Attitude and Preparedness of Healthcare Workers Toward an Outbreak of Ebola Viral Disease
}

\author{
Omobolanle E. Idowu, Adegoke O. Adefolalu, and Dayanithee Chetty
}

\section{ABSTRACT}

\begin{abstract}
Background: Preparedness is key in terms of the healthcare system capacity to react appropriately to an outbreak of any infectious disease in epidemic proportion. Following the Ebola viral disease outbreak that started in West Africa around 2014, which subsequently spread to the DR Congo, with high mortality rates largely attributed to unpreparedness among the healthcare workers, the need for getting all stakeholders involved in healthcare services to be prepared for possible disease outbreaks can never be overemphasized and has since been recognized in many countries including South Africa. This study was conducted to describe the knowledge, attitude and preparedness of healthcare workers towards a possible outbreak of Ebola viral disease at a large private health institution in Pretoria.
\end{abstract}

Method: We conducted a cross-sectional study using self-administered questionnaires among a conveniently sampled 150 healthcare workers at a large private hospital. A total of 133 valid questionnaires were collected (response rate $=89 \%$ ). The data was analyzed with SPSS and results presented using percentages, proportions, and frequency tables.

Results: The finding revealed more female $(93 \%)$ than male, modal agegroup was 31-40yr (37\%), and most were African (89\%). The perceived lack clinical experts who could manage EVD and the fear of contracting the disease were the main concerns of these health professionals. Majority of the participants demonstrated only basic knowledge of EVD but acknowledged the readiness of the nation's health system to deal with any outbreaks.

Conclusion: The participants' insufficient understanding of some aspects of the EVD showed the gaps in their knowledge and the apparent unpreparedness for possible Ebola outbreaks. Therefore, there is need for further education and training among the healthcare workers about how EVD is transmitted and the appropriate measures of disease control and prevention applicable to Ebola virus disease.

Keywords: Ebola virus, disease outbreak, healthcare workers, level of preparedness.

\section{INTRODUCTION}

The Ebola virus disease (EVD) is an acute febrile illness caused by the Ebola virus, a member of the Filovirida family. The virus is mainly transmitted from human-to-humans by close contact with an infected person through body secretions, blood, organ or other bodily fluids, or from infected animals such as fruit bats (which is the primary host), monkeys, gorillas, forest antelope and chimpanzees [1]-[3]. These animals are found in the forests as sick or dead animals or are consumed by humans who eat "bush meat" [1]. Ebola virus is highly virulent and extremely fatal, with a mortality rate of close to $52 \%$ in humans and non-human primates [2][3]. The disease was first discovered more than forty-five years ago around a small village called Ebola in the Democratic Republic of Congo (then Zaire) where the first major outbreak occurred, and from where the disease received its name "Ebola virus" [2]-[3].
Submitted : June 2, 2021

Published. : June 30, 2021

ISSN: 2593-8339

DOI: $10.24018 /$ ejmed.2021.3.3.911

\section{O. E. Idowu}

Department of Nursing Sciences, School of Health Care Sciences, Sefako Makgatho Health Sciences University, Pretoria, South Africa.

(e-mail: bolaridowu@ ${ }^{\circledR}$ rocketmail.com) A. O. Adefolalu*

School of Medicine, Sefako Makgatho Health Sciences University, Pretoria, South Africa.

(e-mail: adegoke.adefolalu@ ${ }^{\circledR}$ smu.ac.za) D. Chetty

Department of Nursing Sciences, School of Health Care Sciences, Sefako Makgatho Health Sciences University,

(e-mail: dayanithee.chetty ${ }^{\circledR}$ smu.ac.za)

*Corresponding Author Pretoria, South Africa.

Since the discovery of the virus in early 1970s there have been several outbreaks of Ebola viral disease in many parts of the world including sub-Saharan Africa, although the patterns of the outbreaks over the years have been somehow periodical in nature, the common threads with all these incidents is that geographically, low-income nations have had more outbreaks compared to high-income nations and there is usually high case fatality rates associated with every outbreak. One of such deadly Ebola outbreaks happened around early 2014 in some parts of West Africa and certain rain forests in Central Africa. The affected countries included Guinea, Liberia, Sierra Leone and Nigeria, to date it remains one of the deadliest outbreaks in recent times. According to the WHO, the number of confirmed Ebola cases reported during this outbreak was about 2127 and 1145 deaths [1], [4]. Reports from WHO revealed that between the year 2014 to 2015, there were several healthcare workers among the people reported to have been infected with Ebola and many 
of them died as a result of the disease, especially in parts of West and Central Africa [4].

The management of an extremely infectious disease like EVD often involves a multidisciplinary approach, whereby the infected individuals are cared for at designated treatment centers equipped with specialized infrastructures and managed by qualified clinicians. The standard operating procedure required that all the healthcare workers $(\mathrm{HCW})$ involved in the management of confirmed cases of Ebola at these centers should practice strict infection control measures at all times, including the use of personal protective equipment (PPE) [5]. The severity of the 2014 Ebola viral disease outbreak and the rate at which it rapidly spread across several West African nations speaks volume about the state of preparedness of these countries' existing health systems in responding to the outbreak. Therefore, the need for all the relevant stakeholders involved in the delivery of healthcare services within the health systems in sub-Saharan Africa to be proactive instead of reactive can never be overemphasized [3]. Decision makers in health care services should ensure their workers are prepared for a possible disease outbreak at all times. The situation of Ebola outbreak in some West African states is not unique in itself, it is very common to find poor preparation for disease Outbreak among healthcare workers of most developing countries. Research has shown that the state of readiness of health systems in many low-income nations in preparation for disease outbreaks are often non-existent. In most instances, adequate resources for emergency response to epidemics are not budgeted for [3]- [5].

According to multiple reports, the major reason for the rapid spread of Ebola virus in the affected West African states during the 2014 outbreak was the free movement of infected people throughout the region without any form of border restrictions, especially the movement of people across the porous land borders within some of the neighboring West African states [2]-[5]. In addition to this, there is strong evidence to suggest that other factors such as the level of preparedness of the health system to deal with epidemics, knowledge and attitude of health care workers towards disease outbreaks, and availability of resources were all contributing factors to the rapid spread and attending high mortality [1]-[5]. In a particular report the lack of adequate personal protective equipment (PPE) was reported as the main reason for the high morbidity and mortality associated with the 2014 Ebola outbreak among the general public [1]. Furthermore, the morbidity and mortality rates recorded among the healthcare workers are strongly linked with the lack of adequate PPE, as well as the ill-preparedness of the few HCW available. All the above-mentioned factors combined together and resulted in the increased spread of the disease and its associated mortality [1]. Therefore, the premature deaths recorded among the healthcare workers tasked with the responsibility of caring for the sick and infirmed during the Ebola epidemic occurred primarily because these healthcare workers were vulnerably exposed due to inadequate resources, most especially the lack of PPEs, and the poor preparation for disease outbreak of that magnitude by their health systems.

Despite the increased understanding of the transmission of the Ebola virus, and the availability of guidelines by the World Health Organization (WHO) and other agencies, studies have shown that the majority of the health care workers do not always use PPE. This has made the nosocomial transmission of Ebola to be a major cause of morbidity and mortality among health care workers. Some of the reason being advanced for this lack of compliance include lack of appropriate equipment and protective clothing, leading to occupational exposure to infections [6]. Although Viral Haemorrhagic Fevers (VHF) appear to occur less frequently in South Africa as there is no record of such an outbreak, it has the potential to cause substantial outbreaks, especially in within healthcare facilities. The country remains at risk for experiencing outbreaks, as the Crimean-Congo Haemorrhagic Fever and the Rift Valley Fever is prevalent in South Africa [7]. Several neighbouring countries are also at risk for VHFs, due to increased local and international travel and trade. The increase in number of severely ill patients being transferred from other tropical African countries seeking medical attention in South Africa has also increased the risk of possible outbreaks of Lassa, Marburg, and Ebola Haemorrhagic fevers [8], [9].

In light of the above, it became obvious to all stakeholders that South Africa health system was vulnerable to epidemic happening in neighbouring countries, especially the southern African countries. The National Department of Health subsequently mandated all healthcare institutions to have a written policy in place aim at curbing any potential spread through importation, of any disease outbreak in South Africa. This policy was driven by the then minister of health who strongly believe such policy would assist in getting healthcare institutions prepared for potential outbreak, thereby reducing the possible spread of such infections in the future. Some of the provisos of this policy is that health care workers are required to maintain high standards of infection control measures at all times as a means of preventing occupational exposure, and all the health care facilities should introduce measures and plans to manage the specific disease-causing epidemic [9].

The severity of an Ebola outbreak cannot be overemphasized. The EVD outbreak of 2014 and rapid spread of disease in epidemic proportion in West Africa revealed poor preparedness to manage VHFs. During this time, several HCWs contracted the disease through nosocomial transmission, it was estimated that $78 \%$ of Sierra Leone healthcare workers reportedly suffered exposure to body fluids of confirmed EVD patients [10], and the WHO reported that approximately $60 \%$ of the infected healthcare workers eventually resulted in death [1]. This confirms the high risk faced by HCWs during EVD outbreak, and further underscores the need to strengthening the health system regarding its preparedness for outbreaks and how to reduce the possibility of nosocomial transmission of Ebola fever. To achieve these, one expects that all the HCWs have access to the necessary information about Ebola-related diseases and have adequate knowledge required of their status in prevention and control of EVD. Although, at the time of conducting this research no confirmed case of EVD had been reported in any of the South African healthcare institutions, very few studies on awareness of the EVD have been conducted [11]. In light of the possible outbreak of EVD in 
South Africa, there is a need to assess the knowledge and preparedness of the healthcare professionals who are the "frontline HCWs" tasked with the responsibility of providing healthcare services in the hospital setting. Hence, this current study aimed to describe the knowledge, attitude and preparedness of HCW towards EVD at a large private hospital in Pretoria, South Africa. The study was conducted to assess the state of preparedness among the healthcare workers for a possible outbreak of EVD within the hospital setting.

\section{METHODS}

The study employed quantitative research method in which a cross-sectional research design was used to collect data from the participants. The study setting was a private hospital in Central Pretoria which is one of the largest private hospitals in the city, offering 24-hour general and specialized health services for adults and children. It receives referrals from doctors in private practice and also from public hospitals, including walk-ins and self-referred patients. The rationale for using the hospital as the study setting stemmed from its big size and the large number of patients seen at the institution. In addition, the hospital had several international patients who travelled down to South Africa for specialized medical treatment, and many of the hospital registered patients were immigrants from various sub-Saharan countries. Therefore, should there be any outbreak of an epidemic disease in the neighbouring countries, the risk of acquiring and transmission of such disease in nosocomial fashion would be higher in places such as the study setting of the current research.

A total of 133 participants were conveniently sampled from a target population consisting of 226 healthcare workers, mainly professional nurses, enrolled nurses, auxiliary nursing assistants, student nurses and care workers. The study was conducted between January to December 2015, data collection was done using self-administered questionnaire. The questionnaire was written in English and made up of four sections. The first section dealt with sociodemographic characteristics of the participants. The second sections asked the participant about their knowledge about Ebola Virus Disease, while third and fourth section asked the participant about their knowledge about control of Ebola Virus Disease and their attitude towards the control of Ebola Virus Disease. Ethical clearance was obtained from the Sefako Makgatho Health Sciences University Research \& Ethics Committee (SMUREC/H/120/2018: PG).

Furthermore, permission to conduct the study was obtained from the hospital management, and written informed consent was obtained from all the participants before commencing the study. SPSS version 20.0 (IBM, Armonk, NY) was used for statistical analyses. Significance was assessed using the ChiSquared test. A P value $<0.05$ was considered to indicate statistical significance.

\section{RESULTS}

The demographics characteristics of the 133 participants are shown in Table I below. It indicates that most of the participants fell into age-group of 31-40 yrs (37\%), and almost all the participants were females $(93 \%)$. The registered nurses made up the largest part of the sample, accounting for $39 \%$ of the total participants, this was followed by the enrolled auxiliary nurses with $26 \%$, while the student nurses and care workers represent the remaining $10 \%$. The majority $(73 \%)$ of the participants had tertiary education, while African race account for nearly all (90\%) of the participants

TABLE I: DEMOGRAPHIC CHARACTERISTICS OF THE PARTICIPANTS

\begin{tabular}{clcc}
\multicolumn{1}{c}{$(\mathrm{N}=133)$} & \multicolumn{1}{c}{ Variables } & Freq. & $\%$ \\
\hline Category & Female & 124 & $93 \%$ \\
& Male & 9 & $7 \%$ \\
Age-group (years) & Total & 133 & $100 \%$ \\
& $20-30$ & 30 & $23 \%$ \\
& $31-40$ & 49 & $37 \%$ \\
& $41-50$ & 34 & $26 \%$ \\
Professional category & $51-60$ & 20 & $15 \%$ \\
& Total & 133 & $100 \%$ \\
& Registered nurse & 52 & $39 \%$ \\
& Enrolled nurse & 33 & $25 \%$ \\
& Enrolled nurse auxiliary & 34 & $26 \%$ \\
& Student nurse & 7 & $5 \%$ \\
& Care worker & 7 & $5 \%$ \\
& Total & 133 & $100 \%$ \\
& White & 12 & $9 \%$ \\
& Indian & 1 & $1 \%$ \\
& African & 118 & $89 \%$ \\
& Coloured & 2 & $2 \%$ \\
& Total & 133 & $100 \%$ \\
& Matric & 30 & $22.5 \%$ \\
& Tertiary & 97 & $72.9 \%$ \\
& Other not specified & 5 & $3.6 \%$ \\
& Other: care worker & 1 & $1 \%$ \\
& Total & 133 & $100 \%$ \\
\hline \multirow{5}{*}{ Education level } & & &
\end{tabular}

The following figures below depicts the questions and responses from the participants about their knowledge of Ebola Virus Disease.

Heard about the Ebola disease and training received

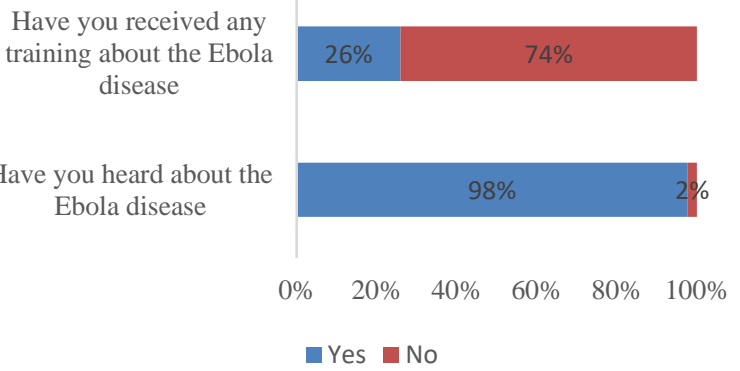

Fig. 1a. Awareness of Ebola Disease and Training Received.

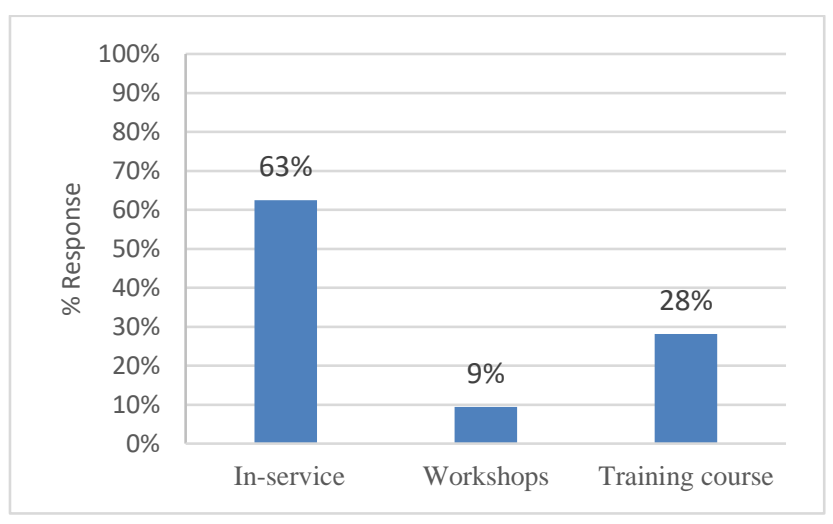

Fig. 1b: Type of Training Received. 


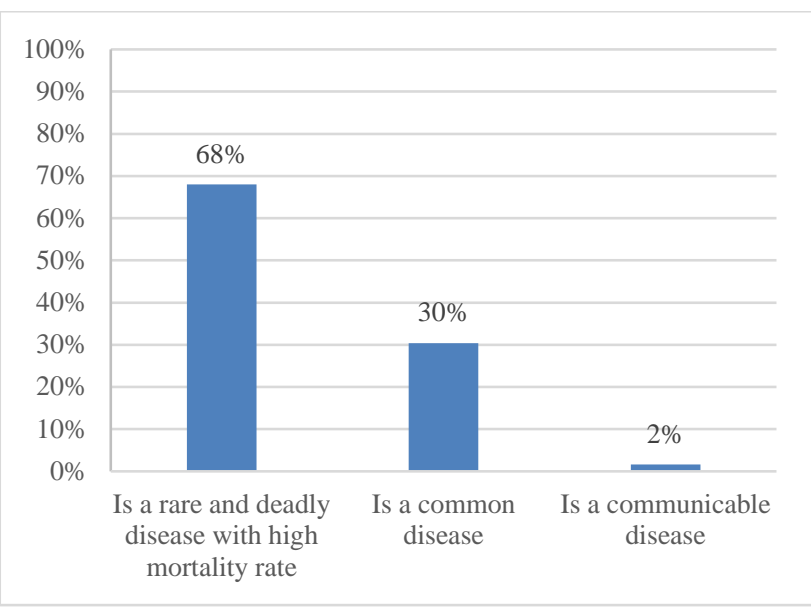

Fig. 1c. Definition of Ebola Disease.

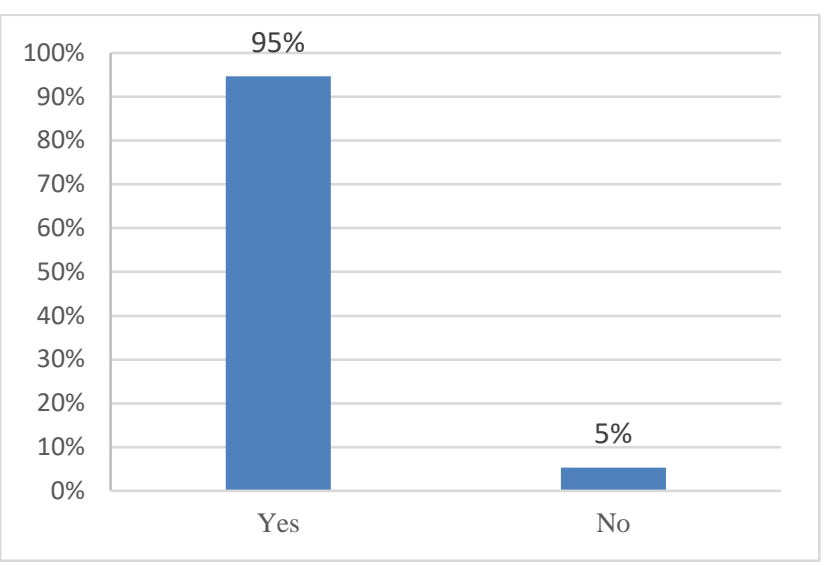

Fig. 1d. Is Ebola Viral Disease a Global Health Concern?

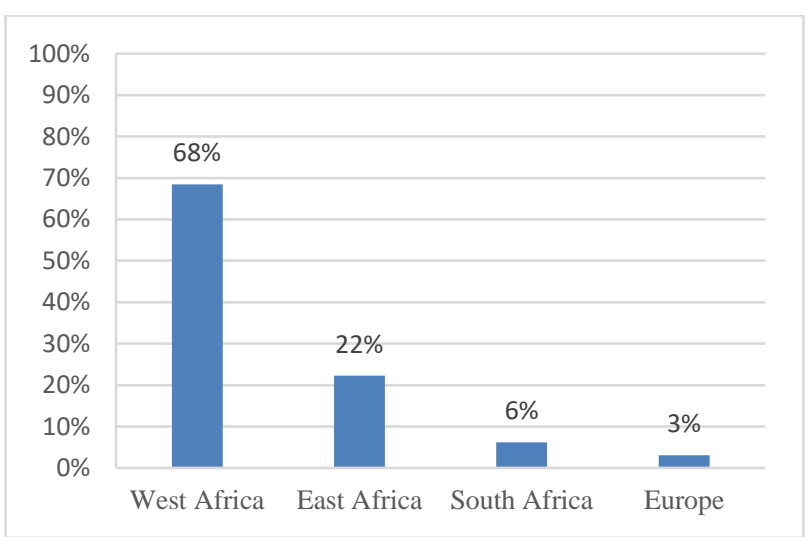

Fig. 1e. Latest Outbreak of Ebola Viral Disease.

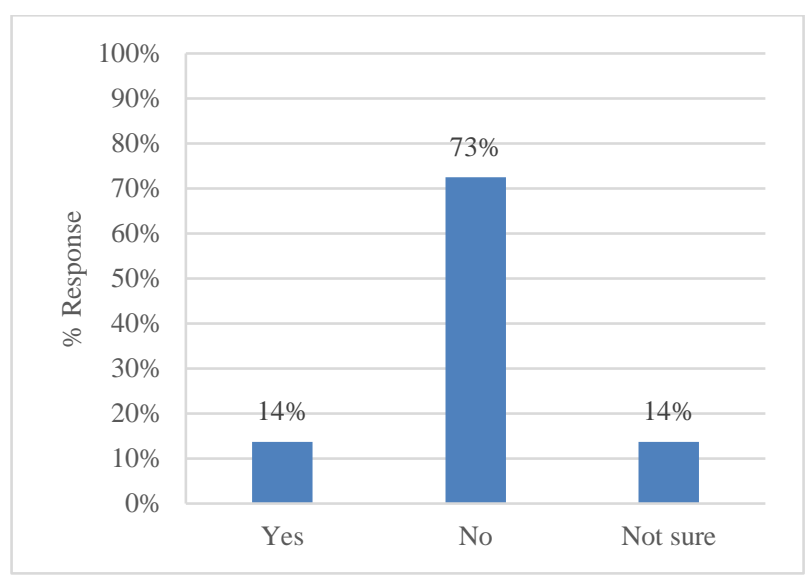

Fig. 1f.: Safe to Use the Same Toilet with Ebola Patients?

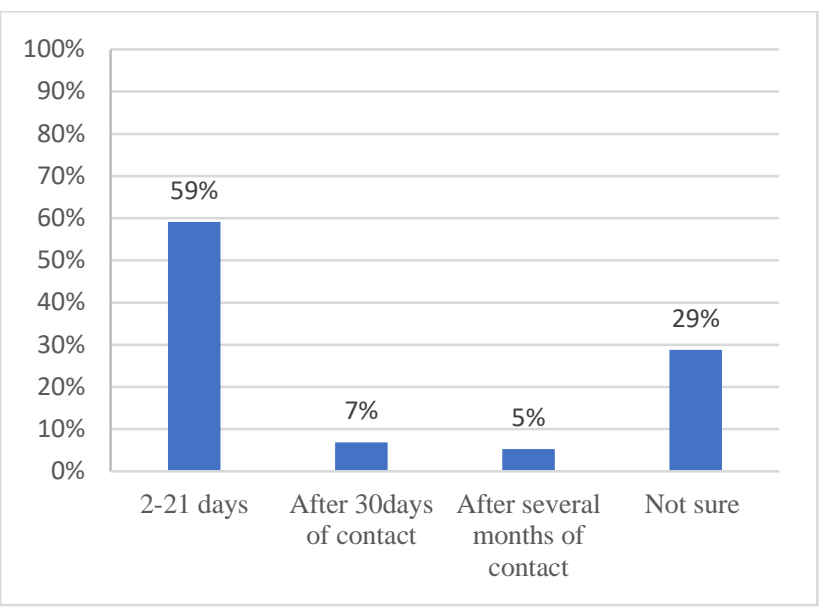

Fig. 1g. What Is the Incubation Period of The Ebola Disease?

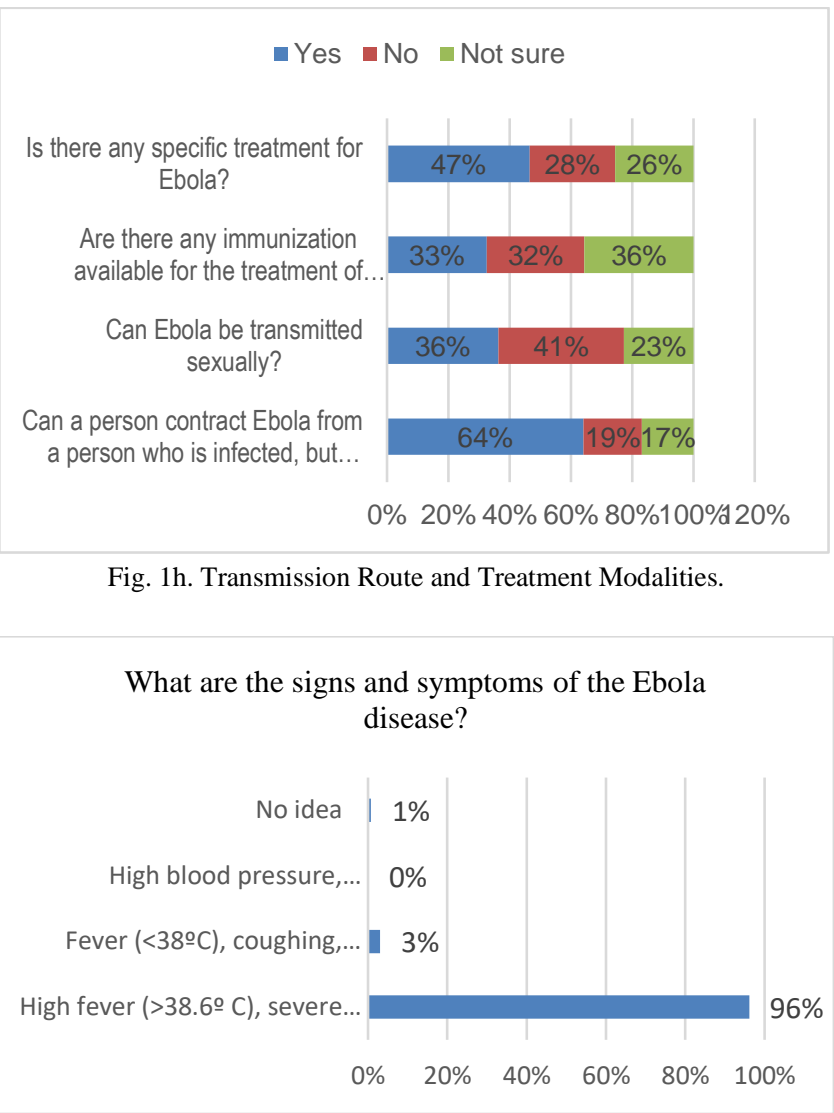

Fig. 1i. Signs and Symptoms of The Ebola Viral Disease.

\section{A. Participants' Knowledge of Ebola Virus Diseases}

The results showed that nearly all of the participants (98\%) have heard about the Ebola Disease, only few (26\%) of the respondents have received training on the disease, and this was done mainly through in-service training organized by the employers for staff members. The majority $(68 \%)$ of the participants correctly indicated that Ebola was a rare and deadly disease associated with high mortality rate, and almost all of them (95\%) agreed that EVD was a global health concern but about two-thirds of the participants (68\%) knew where the latest outbreak of Ebola epidemic had taken place. When asked about sharing of toilet with confirmed Ebola patient, majority (73\%) of the participants correctly indicated that it was not safe to use the same toilet with patients suffering from Ebola viral disease because of the possibility of body secretions. When asked about the incubation period of EVD, more than half (59\%) indicated that the incubation 
period of the Ebola disease is between 2 to 21 days. When asked about Ebola transmission, majority (64\%) responded that a person can contract Ebola from a person who is infected but does not have any symptoms. In addition, about one-third believed that Ebola virus can be transmitted sexually (36\%), and that there was immunization available for the disease (33\%), whereas nearly half $(47 \%)$ of participants answered that there was specific treatment for Ebola. Almost all of them $(96 \%)$ correctly identified the clinical features of EBV.

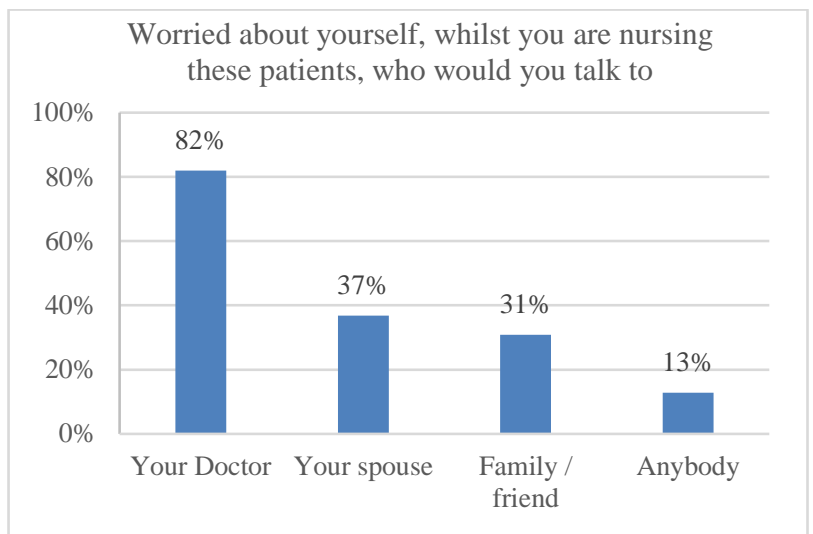

Fig. 2. Participants' Preference in Terms of Communication.

\section{B. Participants' Attitude towards Ebola Virus Diseases}

When asked about their feelings when nursing Ebola patients, many of them reported being nervousness $(36 \%)$, and fearful (35\%), when providing services for a patient who was diagnosed with the Ebola disease. In terms of their concerns, the lack of relevant skills to treat Ebola in South Africa was their main concern (35\%), this was followed by fear of contracting the disease $(50 \%)$ themselves. Almost all the participants $(82 \%)$ will talk to their doctor about their worries whilst nursing Ebola patients. The majority (96\%) of the participants correctly identified hand hygiene as the basic tool in preventing transmission of Ebola among healthcare workers. Furthermore, majority agreed that frequent hand washing (92\%), avoiding contact with blood and bodily fluids of sick persons (89\%), and non-handling of potentially contaminated objects from Ebola-infected persons (83\%), are measures that could be used in protecting oneself from being infected. When asked about touching the dead bodies of Ebola patients, majority $(71 \%)$ indicated it was contraindicated, although almost all the participants (92\%) indicated that they would seek immediate medical care should they develop symptoms of EVD, half of the participants $(50 \%)$ said they would avoid going to hospitals where Ebola patients are being managed. In terms of the preparedness of private hospitals towards outbreak of Ebola disease and likelihood of the disease spreading in South Africa, majority $(80 \%)$ of the participants opined that it was likely for Ebola virus to spread to South Africa, and about half $(48 \%)$ felt that South African private hospitals were ready to cater for such patients should it become necessary.

\section{DISCUSSION}

The participants in this study demonstrated adequate knowledge indicating that they understood the definition of Ebola virus and that the disease is a global health concern.
Most of them were also aware of the current situation globally in terms of where the last outbreak of Ebola virus disease epidemic occurred. This is similar to findings of previous studies in which most of the participants showed basic understanding of EVD and that it is of global public health concern [12], [13]. Although majority of the participants correctly identified the mode of transmission of Ebola viral disease which is mainly through person to person, in particular the direct contact with blood and other bodily fluids of the sick patients. This is in contrast to a 2015 study that found most of their participants not able to correctly identify the common mode of Ebola transmission [14]. Moreover, another study revealed some significant differences in the knowledge of doctors and allied health care providers regarding the modes of transmission and clinical manifestations [15].

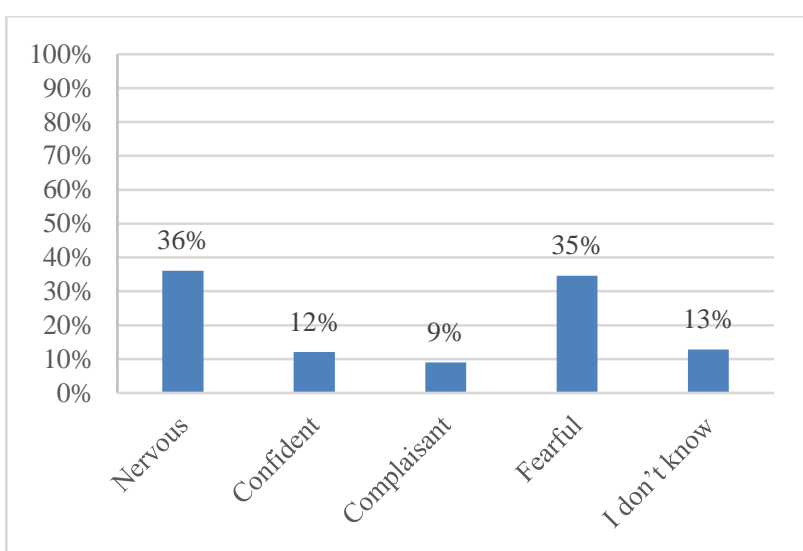

Fig. 3. Participants Feelings When Caring for Ebola Virus Patient..

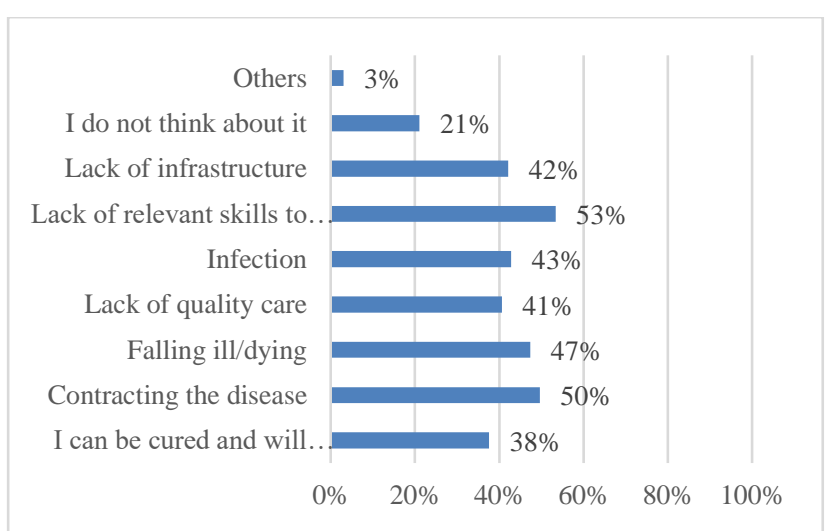

Fig. 4. Participants Main Concern About Ebola Viral Disease.

It appeared the participants are well versed on how the Ebola disease outbreak usually starts, as revealed by their responses. Incubation period of two to 21 days of the Ebola disease was correctly answered by majority of the participants, indicating that most of them have good knowledge on the clinical aspect of the EVD. This finding is very important; the virus has an incubation period of between two and 21 days from the onset of presenting symptoms. This knowledge of incubation period is useful during the implementation of prevention and control measures to combat EVD. In addition, previous research has shown that in line with EVD's incubation period, therefore, the quarantine must last up to 21 days [4], [16]. The signs and symptoms of EVD was answered correctly, which showed that the respondents are fully aware of the signs and 
symptoms of EVD, as most of the respondents correctly indicated the signs and symptoms of the Ebola disease. One earlier study revealed that a lot of people were not confident with Ebola symptom identification [14]. The lack of relevant skills to treat Ebola in South Africa contributed significantly to the attitude of the participants in this study, as majority are worried that EVD is likely to spread within the hospitals which they asserted were not well prepared for the patients. It is worthy to note that majority of the participants were confident that South African private hospitals could cater for patients who are admitted with the Ebola virus if the health care workers are given adequate training and equipment. The fact that some of the participants reported being nervous and fearful while caring for patients are indications that South African private hospitals seemed not to be ready should there be an outbreak of EVD that require patients to be admitted into these hospitals.

\section{CONCLUSION}

The results of this study show that the fact that the Ebola virus disease (EVD) is a global health issue is widely known in South Africa health care workers. The signs and symptoms of the virus are also understood by the health care workers. The attitude and their preparedness of the health care workers are based on the provision of protective equipment by the management of the hospital and special training. It is recommended that more training be provided for identified staff and where identified, adequate PPE should be made available to enable healthcare workers perform their duties without the fear of contracting infectious diseases.

\section{ACKNOWLEDGMENT}

The authors wish to thank the management and staff of Mediclinic Medforum Hospital in Pretoria for their assistance in conducting this research.

\section{REFERENCES}

[1] World Health Organization. WHO: Ebola Response Roadmap Situation Report, World Health Organization. 2014. Available: http://www.who.int/csr/disease/ebola/situation-reports/en.

[2] E. Sterk, Filovirus Haemorrhagic Fever Guidelines, Barcelona: Medecins Sans Frontieres Operational Center Barcelona-Spain; pp. 1-138, 2008. Available:

https://www.ghdonline.org/uploads/MSF_Ebola_2008.pdf.

[3] S. Kishore, and R. Singh, Ebola Virus Disease-An Update. Indian Journal of Community Health, vol. 26, no. 4, pp. 443-445. Dec. 2014.

[4] A. Jarrett, Ebola: A practice summary for nurse practitioners. The Journal for Nurse Practitioners, vol. 11, no. 1, pp. 16-26. Jan. 2015.

[5] O.E. Amoran, and O.O. Onwube, Infection control and practice of standard precautions among healthcare workers in northern Nigeria. Journal of Global Infectious Diseases, vol. 5, no. 4, pp. 156-163. Oct. 2013.

[6] P. Shears, and T.J.D. O' Dempsey, Ebola virus disease in Africa: epidemiology and nosocomial transmission. Journal of Hospital Infection, vol. 90, no. 1, pp. 1-9. Jan. 2015.

[7] P.J. Van Vuren, J. Weyer, A. Kemp, C. le Roux, P. Leman, A. Grobbelaar, P. Lekhuleni, V. Dermaux-Msimang, B. Archer, J. Thomas, L. Blumberg, and J. Paweska Viral Haemorrhagic fever outbreaks, South Africa, 2011. Comm. Dis. Surveill. Bull, vol. 10, no. 1, pp. 1-5. April. 2012. Available: https://www.nicd.ac.za/assets/files/CommDisBull\%2010(1)April\%202012.pdf.

[8] M.A. Daw, and A. El-Bouzedi, Viral haemorrhagic fever in North Africa; an evolving emergency. Journal of Clinical \& Experimental Pathology, vol. 5, no. 215, pp. 2161-0681. Feb. 2015.

[9] Department of Health. Health budget vote speech by the Minister of Health. Pretoria: Government of Republic of South Africa. 2014.

[10] A.C. Dunn, T.A. Walker, J. Redd, D. Sugerman, J. McFadden, T. Singh, and P.H. Kilmarx, Nosocomial transmission of Ebola virus disease on paediatrics and maternity wards: Bombali and Tonkolili, Sierra Leone, 2014. American Journal of Infection Control, vol. 44, no. 3, pp. 269-272. March. 2016.

[11] K. Changula, M. Kajihara, A.S. Mweene, A. Takada, Ebola and Marburg virus diseases in Africa: increased risk of outbreaks in previously unaffected areas? Microbiology and Immunology, vol. 58, no. 9 , pp. 483-491. Sept. 2014.

[12] A. Hisam, and M.N Rana. Knowledge and attitude regarding Ebola virus disease among medical students of Rawalpindi: A preventable threat not yet confronted. Pakistan Journal Of Medical Sciences, vol. 32, no. 4, pp. 1015-1019. Jul-Aug 2016.

[13] Y. Liu, T. Li, Y. Liu, Y. Hao, Y. J. Wang, Y. Wu, \& K. Zhao. Development of HIV-1 vaccines based on replication competent Tiantan vaccinia vector in China. The Lancet, vol. 386, suppl.10. 2015

[14] M. Kobayashi, K.D. Beer, A. Bjork, K. Chatham-Stephens, C.C. Cherry, C.C., Arzoaquoi, S., Frank, W., Kumeh, O., Sieka, J., Yeiah, A. \& Painter, J.E. "Community knowledge, attitudes, and practices regarding Ebola virus disease-five counties, Liberia, SeptemberOctober 2014," MMWR. Morbidity And Mortality Weekly Report, 64(26), 714. 2015.

[15] M. M. Alfaki, A. M. Salih, D. A. L. Elhuda, and M. S Egail, "Knowledge, attitude, and practice of health care providers toward Ebola virus disease in hotspots in Khartoum and White Nile states, Sudan, 2014," American Journal of Infection Control, vol. 44, no. 1, pp. 20-23. Jan 2016.

[16] L. O Gostin, D. Lucey and A. Phelan, "The Ebola epidemic: a global health emergency," Journal of American Medical Association, vol. 312, no. 11, pp. 1095-1096. Sept. 2014.
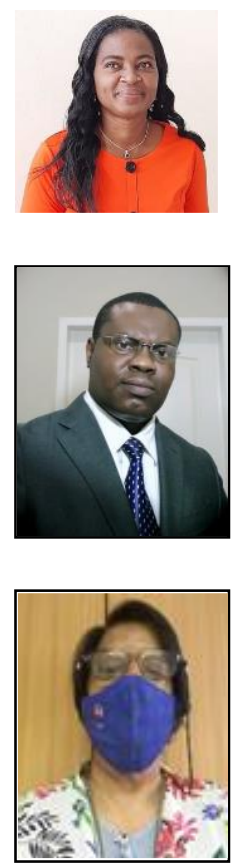

Mrs. Omobolanle E, Idowu, RN, RM, BNSc, MCur, is a postgraduate student at the Department of Nursing Sciences, School of Health Care Sciences, Sefako Makgatho Health Sciences University, Pretoria, South Africa. She currently works as a Professional nurse at Mediclinic Medforum Hospital Pretoria South Africa.

Dr Adegoke O. Adefolalu, $\mathrm{MBChB}, \mathrm{MPH}, \mathrm{PhD}$, FRSPH, is at the School of Medicine, Sefako Makgatho Health Sciences University, South Africa. A Public Health physician with research interests in clinical epidemiology, HIV/AIDS, health systems, health behaviour and health determinants. An astute health advocate, he is involved in mentorship and participates actively in staff development.

Dr Dayanithee Chetty, M Cur: D Cur; is a senior faculty, Assessor and Moderator.at the Department of Nursing Sciences, School of Health Care Sciences, Sefako Makgatho Health Sciences University, Pretoria, South Africa. She has specialization in Advanced Psychiatric Nursing Science; Quantitative and Qualitative Research Methodology. 